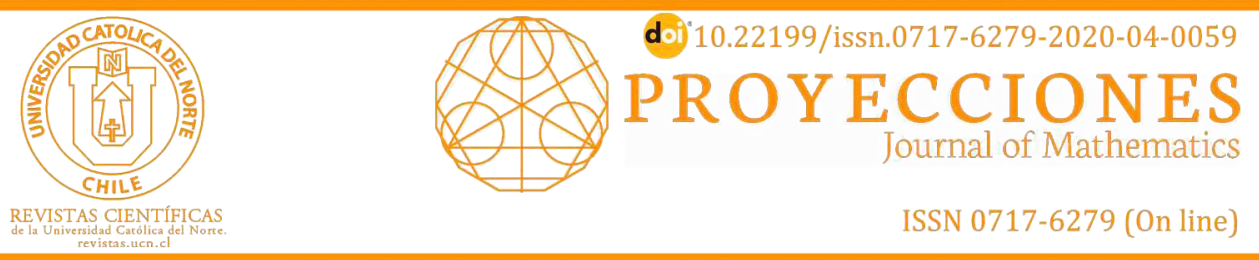

\title{
Modules whose partial endomorphisms have a $\delta$-small kernels
}

\author{
Papa Cheikhou Diop ${ }^{1}$ (i) orcid.org/0000-0003-0628-0018 \\ Abdoul Djibril Diallo ${ }^{2}$ @ orcid.org/0000-0002-1791-0639
}

${ }^{1}$ Université de Thiès, Dépt. de Mathématiques, Thiès, Sénégal.

cheikpapa@gmail.com

${ }^{2}$ Université Cheikh Anta Diop, Dépt. de Mathématiques et Informatique, Dakar, Sénégal.

dialloabdoulaziz58@yahoo.fr

\section{Abstract:}

Let $R$ be a commutative ring and $M$ a unital $R$-module. $A$ submodule $N$ is said to be $\delta$-small, if whenever $N+L=M$ with $M / L$ is singular, we have $L=$ $M$. $M$ is called $\delta$-small monoform if any of its partial endomorphism has $\delta$-small kernel. In this paper, we introduce the concept of $\delta$-small monoform modules as a generalization of monoform modules and give some of their properties, examples and characterizations.

Keywords: Small submodules; $\delta$-small submodules; Monoform modules; $\delta$-small monoform modules; Artinian principal ideal ring.

MSC (2020): 16D10, 16D40, 16P40, 16L30.

\section{Cite this article as (IEEE citation style):}

P. C. Diop and A. D. Diallo, "Modules whose partial endomorphisms have a $\delta$-small kernels", Proyecciones (Antofagasta, On line), vol. 39, no. 4, pp. 945-962, Aug. 2020, doi: 10.22199/issn.0717-6279-2020-04-0059.

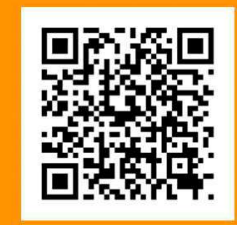

Article copyright: (C) 2020 Papa Cheikhou Diop and Abdoul Djibril Diallo. This is an open access article distributed under the terms of the Creative Commons License, which permits unrestricted use and distribution provided the original author and source are credited. 


\section{Introduction}

Throughout all rings are associative, commutative with identity and all modules are unitary $R$-module. The concept monoform module is appeared in [12], where an $R$-module $M$ is called monoform if for each nonzero submodule $N$ of $M$ and for each $f \in \operatorname{Hom}(N, M), f \neq 0$ implies $\operatorname{Ker} f=\{0\}$. In [6], M. A. Inaam Hadi and al. introduced and studied the concept of small monoform, where a module $M$ is called module small monoform if for each nonzero submodule $N$ of $M$ and for each nonzero $f \in \operatorname{Hom}(N, M)$, $\operatorname{Kerf}$ is small in $N$. A submodule $L$ of a module $M$ is called essential submodule of $M$, if $L \cap K \neq\{0\}$ for any nonzero submodule $K$ of $M$. A submodule $N$ of a module $M$ is called e-small submodule of $M$ if, for any essential submodule $L$ of $M, N+L=M$ implies $L=M$. Recently in [5], A. D. Diallo and al introduced and studied the concept of $e$-small monoform, where a module $M$ is called module $e$-small monoform if for each nonzero submodule $N$ of $M$ and for each nonzero $f \in \operatorname{Hom}(N, M), \operatorname{Ker} f$ is $e$-small in $N$. In [13], Zhou. Y introduced and studied the concept of $\delta$-small submodule, where a submodule $N$ of $M$ is called $\delta$-small $\left(N \ll_{\delta} M\right.$, for short) if whenever $N+L=M$ with $M / L$ is singular, then $L=M$. An $R$-module $M$ is called $\delta$-small monoform if any of its partial endomorphism has $\delta$-small kernel.

In this paper, we introduce and study the concept of $\delta$-small monoform module as a new generalization of monoform module and give some of their properties, characterizations and examples. A module $M$ is called compressible if it can be embedded in each of its nonzero submodules. Then, we have the obvious implications:

compressible $\Rightarrow$ monoform $\Rightarrow$ small monoform $\Rightarrow \delta$-small monoform $\Rightarrow e$-small monoform. We will prove later that under certain conditions the properties compressible, monoform, small monoform and $\delta$-small monoform are equivalent.

Morever, we study the relations between $\delta$-small monoform modules and other related modules as nonsingular module, quasi-injective module and fully retractable module.

Our paper is structured as follows: 
In the first section, we give some known results which we will use or cite throughout this paper.

In the second section, we define the $\delta$-small monoform modules. Our aim in this section is to work on the concept of $\delta$-small monoform modules. We also show among others, the following results.

(1) The following statements are equivalent for an $R$-module $M$ :

(a) $M$ is a $\delta$-small monoform module.

(b) For any $0 \neq N \leq M$ and for any nonzero $f \in \operatorname{Hom}_{R}(N, M)$, if there exists $K \leq N$ such that $f(K)=f(N)$ and $\left(K+Z_{2}(N)\right) \leq_{e} N$, then $N=K$. (c) For any $0 \neq N \leq M$ and for any nonzero $f \in \operatorname{Hom}_{R}(N, M)$, Kerf $\subseteq$ $\delta(N)$ and if there exists $K \leq N$ with $f(K)=f(N)$, then there exists a projective $L \leq^{\oplus} N$ such that $L \subseteq K \operatorname{Kerf}$ and $N=K \oplus L$.

Also we have a characterization of small monoform modules.

(2) The following statements are equivalent for an $R$-module $M$ :

(a) $M$ is a small monoform module.

(b) For any $0 \neq N \leq M$ and for any nonzero $f \in \operatorname{Hom}_{R}(N, M)$, Kerf $\subseteq$ $\operatorname{Rad}(N)$ and if there exists $K \leq N$ with $f(K)=f(N)$, then there exists a projective $L \leq^{\oplus} N$ such that $L \subseteq K \operatorname{erf}$ and $N=K \oplus L$.

Finally, we prove the following theorem.

(3) Let $R$ be an artinian principal ideal ring and $M$ be a singular prime $R$-module. Then the following statements are equivalent:

(a) $M$ is a $\delta$-small monoform module.

(b) $M$ is a monoform module.

(c) $\mathrm{M}$ is a small monoform module.

(d) $M$ is a compressible module.

(e) $\mathrm{M}$ is a uniform module.

(f) $M$ is a weakly co-Hopfian module.

\section{Preliminaries}

In this section, we give some known results which we will use or cite throughout this paper.

Definition 2.1. Let $M$ be an $R$-module, put $Z(M)=\left\{m \in M:\right.$ ann $_{R}(m) \leq_{e}$ $R\} . M$ is called nonsingular if $Z(M)=\{0\}$, and singular if $Z(M)=M$. The Goldie torsion submodule $Z_{2}(M)$ of $M$ is defined by $Z(M / Z(M))=$ $Z_{2}(M) / Z(M) . M$ is called Goldie torsion if $M=Z_{2}(M)$. 
Definition 2.2. Let $M$ be an $R$-module and $N \leq M$.

1. $N$ is called small submodule of $M$ ( $N \ll M$, for short) if, for any submodule $L$ of $M, N+L=M$ implies $L=M$

2. A submodule $N$ is called $\delta$-small ( $N \ll_{\delta} M$, for short) if whenever $N+L=M$ and $M / L$ is singular then $L=M$.

Remark 2.3. Each small submodule, or nonsingular semi-simple of a module $M$ is $\delta$-small submodule in $M$. The $\delta$-small submodules of a singular module are small submodules.

Lemma 2.4. ([13], Lemma 1.2)

A submodule $N$ of a module $M$ is $\delta$-small if and only if for all $X \leq M$ : if $X+N=M$, then $M=X \oplus Y$ for a projective semi-simple submodule $Y$ with $Y \leq N$.

Lemma 2.5. ([13], Lemma 1.3)

1. Let $N, K$ and $L$ be submodules of an $R$-module $M$ such that $K \subseteq N$, then $N \ll_{\delta} M$ if and only if $K \ll_{\delta} M$ and $N / K \ll_{\delta} M / K$.

2. If $K \ll_{\delta} M$ and $f: M \longrightarrow M^{\prime}$ is a homomorphism, then $f(K) \ll_{\delta} M^{\prime}$. In particular, if $K \ll_{\delta} M \subseteq M^{\prime}$, then $K \ll_{\delta} M^{\prime}$.

3. Assume that $K_{1} \subseteq M_{1} \subseteq M, K_{2} \subseteq M_{2} \subseteq M$ and $M=M_{1} \oplus M_{2}$, then $K_{1} \oplus K_{2} \ll_{\delta} M_{1} \oplus M_{2}$ if and only $K_{1} \ll_{\delta} M_{1}$ and $K_{2} \ll_{\delta} M_{2}$.

Definition 2.6. Let $\wp$ be the classe of all singular simples modules. For a module $M$, let $\delta(M)=\operatorname{Re}_{M}(\wp)=\cap\{N \subseteq \mid M / N \in \wp\}$ be the rejet in $M$ of $\wp$.

\section{Main results}

In this section, we introduce the notion of $\delta$-small monoform module and obtain some of its basic properties. It is shown that every submodule of a $\delta$-small monoform module inherits the property (Proposition 2.5). Also we characterize the $\delta$-small monoform modules (Proposition 2.9 and Theorem 2.17). Moroever a characterization of small monoform modules is obtained (Proposition 2.22). Also we prove that under certain conditions, the properties compressible, monoform, small monoform, $\delta$-small monoform and weakly co-Hopfian are equivalent (Theorem 2.32).

Definition 3.1. An $R$-module $M$ is called a $\delta$-small monoform if for each nonzero submodule $N$ of $M$ and for each $f \in \operatorname{Hom}(N, M), f \neq 0$ implies $\operatorname{Kerf} \ll_{\delta} N$. 
Example 3.2. Every nonsingular semi-simple module is a $\delta$-small monoform. But the converse is not true in general.

In fact, the $\mathbf{Z}$-module $\mathbf{Z} / 4 \mathbf{Z}$ is a $\delta$-small monoform but it is neither semisimple nor nonsigular.

Example 3.3. Let $M$ be a Noetherian $R$-module. Suppose for any $0 \neq$ $N \leq M$, every proper submodule of $N$ is contained in $\delta(N)$. Then $M$ is a $\delta$-small monoform module.

Remark 3.4. (1) It is clear that every monoform module is a $\delta$-small monoform. But the converse is not true in general, for example $\mathbf{Z} / 4 \mathbf{Z}$ as $\mathbf{Z}$-module is a $\delta$-small monoform but it is not monoform. (2) The homomorphic image of $\delta$-small monoform module is not necessarily $\delta$-small monoform; for example $\mathbf{Z}$ as $\mathbf{Z}$-module is $\delta$-small monoform.

Let $\pi: \mathbf{Z} \longrightarrow \mathbf{Z} / 12 \mathbf{Z}$, where $\pi$ is the natural projection. However $\mathbf{Z} / 12 \mathbf{Z}$ as $\mathbf{Z}$-module is not $\delta$-small monoform because $\overline{0} \neq f=4 \bar{x} \in \operatorname{End}_{\mathbf{Z}}(\mathbf{Z} / 12 \mathbf{Z})$ and

$\operatorname{Kerf}=<\overline{3}>K_{\delta} \mathbf{Z} / 12 \mathbf{Z}$. From this, it follows easily that quotients of $\delta$-small monoform modules need not to be $\delta$-small monoform in general.

Proposition 3.5. Every nonzero submodule of $\delta$-small monoform module is a $\delta$-small monoform.

Proof. Let $M$ be a $\delta$-small monoform $R$-module. For any $K \leq N$, $K \neq\{0\}$, let $f: K \longrightarrow N$ such that $f \neq 0$. Then $i \circ f \neq 0$, where $i$ is the inclusion mapping. Thus, $\operatorname{Ker}(i \circ f) \ll_{e} K$ because $M$ is a $\delta$-small monoform module. But $\operatorname{Ker}(i \circ f)=\operatorname{Kerf}$, hence $\operatorname{Kerf} \ll_{\delta} K$ and so $N$ is a $\delta$-small monoform module.

Remark 3.6. The direct sum of $\delta$-small monoform modules is not necessarily

$\delta$-small monoform module. Consider the following examples:

1. Let $M=\mathbf{Z} / 4 \mathbf{Z} \oplus \mathbf{Z} / 3 \mathbf{Z}$ as $\mathbf{Z}$-module. Each of $\mathbf{Z} / 4 \mathbf{Z}$ and $\mathbf{Z} / 3 \mathbf{Z}$ is a $\delta$-small monoform and $M=\mathbf{Z} / 4 \mathbf{Z} \oplus \mathbf{Z} / 3 \mathbf{Z} \cong \mathbf{Z} / 12 \mathbf{Z}$. However $\mathbf{Z} / 12 \mathbf{Z}$ as $\mathbf{Z}$-module is not $\delta$-small monoform.

2. The $\mathbf{Z}$-module $\mathbf{Z} \oplus \mathbf{Z}$ is not $\delta$-small monoform while $\mathbf{Z}$ is a $\delta$-small monoform $\mathbf{Z}$-module.

Definition 3.7. An R-module $M$ is called fully stable if for each $N \leq M$, $N$ is stable, that is for each homomorphism $f: N \longrightarrow M, f(N) \subseteq N$. 
Theorem 3.8. Let $M$ be a fully stable and $R$-module such that $M=M_{1} \oplus M_{2}$, where $M_{1}$ and $M_{2}$ are two submodules of $M$ and for each $R$-homomorphism $f: N_{1} \oplus N_{2} \longrightarrow M, f \neq 0$ implies $f\left(N_{1}\right) \neq\{0\}$ and $f\left(N_{2}\right) \neq\{0\}$ (i.e $\left.f_{\left.\right|_{N_{1}}} \neq 0, f_{\left.\right|_{N_{2}}} \neq 0\right)$. Then $M_{1}$ and $M_{2}$ are $\delta$-small monoforms if and only if $M$ is a $\delta$-small monoform.

Proof.

$\Rightarrow$ Let $0 \neq N \leq M, f: N \longrightarrow M$ such that $f \neq 0$. Since $M$ is fully stable, every submodule of $M$ is stable. So $N$ is stable. Thus, $N=\left(N \cap M_{1}\right) \oplus$ $\left(N \cap M_{1}\right)$ by [1, Proposition 4.5, P.29].

Consider:

$$
\begin{aligned}
& \left(N \cap M_{1}\right) \stackrel{i_{1}}{\longrightarrow} N \stackrel{f}{\longrightarrow} M \stackrel{p_{1}}{\longrightarrow} M_{1} \\
& \left(N \cap M_{2}\right) \stackrel{i_{2}}{\longrightarrow} N \stackrel{f}{\longrightarrow} M \stackrel{p_{2}}{\longrightarrow} M_{2}
\end{aligned}
$$

where $i_{1}, i_{2}$ are inclusion mappings and $p_{1}, p_{2}$ are projection mappings. Then $p_{1} \circ f \circ i_{1}:\left(N \cap M_{1}\right) \longrightarrow M_{1}$ and $p_{2} \circ f \circ i_{2}:\left(N \cap M_{1}\right) \longrightarrow M_{2}$. Let $N_{1}=N \cap M_{1}$ and $N_{2}=N \cap M_{2}$. By hypothesis, $f_{\left.\right|_{1}} \neq 0$, so there exists $n_{1} \in N \cap M_{1}$,

$n_{1} \neq 0$ and $f\left(n_{1}\right) \neq 0$. Also, $f_{\left.\right|_{N_{2}}} \neq 0$, so there exists $n_{2} \in N \cap M_{2}, n_{2} \neq 0$ and $f\left(n_{2}\right) \neq 0$. On the other hand, $f \circ i_{1}:\left(N \cap M_{1}\right) \longrightarrow M$ implies $f \circ i_{1}\left(n_{1}\right)=f\left(n_{1}\right) \neq 0$ and $f \circ i_{2}:\left(N \cap M_{2}\right) \longrightarrow M$ implies $f \circ i_{2}\left(n_{2}\right)=$ $f\left(n_{2}\right) \neq 0$. Thus,

$f \circ i_{1}\left(N \cap M_{1}\right) \subseteq N \cap M_{1}$ because $N_{1}$ and $N_{2}$ are stable, $f\left(N \cap M_{1}\right) \subseteq N \cap M_{1}$. Similarly $f\left(N \cap M_{2}\right) \subseteq N \cap M_{2}$. Since $f\left(n_{1}\right) \in N \cap M_{1}$ and $f\left(n_{1}\right) \neq 0$, so $p_{1} \circ f \circ i_{1}\left(n_{1}\right)=f\left(n_{1}\right) \neq 0$. Similarly, $p_{2} \circ f \circ i_{2}\left(n_{2}\right)=f\left(n_{2}\right) \neq 0$. Thus $p_{1} \circ f \circ i_{1} \neq 0$ and $p_{2} \circ f \circ i_{2} \neq 0$. Since $M_{1}$ and $M_{2}$ are $\delta$-small monoforms, $\operatorname{Ker}\left(p_{1} \circ f \circ i_{1}\right) \ll_{\delta}\left(N \cap M_{1}\right)$ and $\operatorname{Ker}\left(p_{2} \circ f \circ i_{2}\right) \ll_{\delta}\left(N \cap M_{2}\right)$. Then by Lemma 1.5,

$\operatorname{Ker}\left(p_{1} \circ f \circ i_{1}\right) \oplus \operatorname{Ker}\left(p_{2} \circ f \circ i_{2}\right) \ll_{\delta}\left(N \cap M_{1}\right) \oplus\left(N \cap M_{2}\right)=N$.

Let $x=x_{1}+x_{2} \in K \operatorname{Ker} f$, where $x_{1} \in N \cap M_{1}$ and $x_{2} \in N \cap M_{2}$. So $f\left(x_{1}\right)+f\left(x_{2}\right)=0$. Hence, $f\left(x_{1}\right)=-f\left(x_{2}\right) \in\left(N \cap M_{1}\right) \cap\left(N \cap M_{2}\right)=\{0\}$, so $f\left(x_{1}\right)=0$ and $f\left(x_{2}\right)=0$. So, $p_{1} \circ f \circ i_{1}\left(x_{1}\right)=p_{1} \circ f\left(x_{1}\right)=f\left(x_{1}\right)=0$ and $p_{2} \circ f \circ i_{2}\left(x_{1}\right)=p_{2} \circ f\left(x_{2}\right)=f\left(x_{2}\right)=0$. Hence, $x=x_{1}+x_{2} \in$ $\operatorname{Ker}\left(p_{1} \circ f \circ i_{1}\right) \oplus \operatorname{Ker}\left(p_{2} \circ f \circ i_{2}\right) \ll_{\delta} N$. It follows that $\operatorname{Ker} f \subseteq \operatorname{Ker}\left(p_{1} \circ\right.$ $\left.f \circ i_{1}\right) \oplus \operatorname{Ker}\left(p_{2} \circ f \circ i_{2}\right) \ll_{\delta} N$. Thus by Lemma $1.5, \operatorname{Ker} f \ll_{\delta} N$ and so $M$ is a $\delta$-small monoform.

( $\Leftarrow$ It is clear by Proposition 2.5 . 
Proposition 3.9. Let $M$ be a nonzero Noetherian $R$-module. Then $M$ is a $\delta$-small monoform if and only if every nonzero 3 -generated submodule of $M$ is a $\delta$-small monoform.

Proof. $\Rightarrow$ ) It follows from Proposition 2.5.

$(\Leftarrow$ Suppose that every nonzero 3 -generated submodules is a $\delta$-small monoform. Let $0 \neq N \leq M$ and let $f: N \longrightarrow M$ such that $f \neq 0$. If $\operatorname{Kerf}=\{0\}$, then Kerf $\ll_{\delta} N$. If $K \operatorname{Kerf} \neq\{0\}$, let $x \neq 0$ and $x \in \operatorname{Kerf}$. Let $y \in N$ and let $f(y)=z$. Put $L=R x+R y+R z, L$ is 3-generated submodule of $M$.

By hypothesis, $L$ is a $\delta$-small monoform. Let $H=R x+R y$, and $g=f_{\left.\right|_{H}}$ : $H \longrightarrow L$. Hence $\operatorname{Kerg} \ll_{\delta} H \leq N$ because $L$ is a $\delta$-small monoform. But $x \in \operatorname{Kerg}$, so

$\left\langle x>\subseteq \operatorname{Kerg} \ll_{\delta} N\right.$. Since $M$ is Noetherian, $\operatorname{Kerf}$ is finitely generated. Hence $\operatorname{Kerf}=\sum_{i=1}^{n} R x_{i}$, for some $x_{1}, x_{2}, \ldots, x_{n} \in M$. We have $<x_{i}>\ll_{\delta}$ $N$ for each $i=1, \ldots, n$. Thus by Lemma $1.5, \operatorname{Kerf}=\sum_{i=1}^{n} R x_{i} \ll_{\delta} N$. So $M$ is a $\delta$-small monoform $R$-module.

Recall that a module is called weakly co-Hopfian if, for any injective endomorphism $f$ of $M, f(M) \leq_{e} M$.

Corollary 3.10. Let $R$ be an artinian principal ideal ring. Then the following statements are equivalent for a nonzero weakly co-Hopfian $R$-module $M$ :

(1) $M$ is a $\delta$-small monoform module.

(2) Every nonzero 3-generated submodule of $M$ is a $\delta$-small monoform.

\section{Proof.}

Suppose $M$ is a weakly co-Hopfian $R$-module. Then, according to Theorem 3.8 in [3], $M$ is a finitely generated module. Hence $M$ is a Noetherian module because $R$ is an artinian ring. Therefore, in view of Proposition 2.9 , the result is obtained.

Definition 3.11. An $R$-module $M$ is called small monoform if for each nonzero submodule $N$ of $M$ and for each $f \in \operatorname{Hom}(N, M), f \neq 0$ implies $\operatorname{Kerf} \ll N$.

Remark 3.12. It is clear that every small monoform module is a $\delta$-small monoform. But the converse is not true in general; for example let $R$ be 
an artinian semi-simple ring and $M$ be a nonzero $R$-module which is not of finite length. Cleary, $M$ is a $\delta$-small monoform module. But it is not small monoform.

In fact, $M$ is a nonsingular semi-simple module and so it is a $\delta$-small monoform $R$-module. Now, let $f: M \longrightarrow M$ be a nonzero endomorphism. Then, $\operatorname{Kerf} \neq\{0\}$. Since $M$ is a semi-simple module, $\operatorname{Ker} \nless M$. Therefore, $M$ is not small monoform.

Definition 3.13. (1) A submodule $N$ of a module $M$ is called e-small $\left(N \ll_{e} M\right.$, for short) if, for any essential submodule $L$ of $M, N+L=M$ implies $L=M$.

(2) An $R$-module $M$ is called e-small monoform if for each nonzero submodule $N$ of $M$ and for each $f \in \operatorname{Hom}(N, M), f \neq 0$ implies Kerf $\ll_{e} N$.

Remark 3.14. It is clear that every $\delta$-small monoform module is an $e$ small monoform. But the converse is not true in general; for example $\mathbf{Z} / 6 \mathbf{Z}$ is e-small monoform as $\mathbf{Z}$-module. But it is not $\delta$-small monoform. So, accordind to Remark, it is easily seen that e-small monoform modules need not be $\delta$-small monoform or small monoform in general.

Proposition 3.15. Every nonsingular e-small monoform $R$-module is a $\delta$-small monoform module.

Proof.

Suppose $M$ is a nonsingular $e$-small monoform module. Let $0 \neq N \leq M$ and $f: N \longrightarrow M$ be a nonzero homomorphism. Suppose $\operatorname{Ker} f+K=N$ with $N / K$ singular for some $K \leq N$. Since $M$ is a nonsingular module, $N$ is also a nonsingular module. Thus, $K \leq_{e} N$. Since $M$ is assumed $e$-small monoform, $K=N$. Therefore, $M$ is a $\delta$-small monoform module.

Lemma 3.16. ([5], Proposition 2.24)

Let $M$ be a uniform $R$-module. Then $M$ is small monoform if and only if $M$ is an e-small monoform.

Theorem 3.17. The following statements are equivalent for an $R$-module $M$ :

(1) $M$ is a $\delta$-small monoform module.

(2) For any $0 \neq N \leq M$ and for any nonzero $f \in \operatorname{Hom}_{R}(N, M)$, if there exists $K \leq N$ such that $f(K)=f(N)$ and $\left(K+Z_{2}(N)\right) \leq_{e} N$, then $N=K$. 
(3) For any $0 \neq N \leq M$ and for any nonzero $f \in \operatorname{Hom}_{R}(N, M)$, if there exists $K \leq N$ such that $\left(\left(K+Z_{2}(N)\right) / Z_{2}(N)\right) \leq_{e} N / Z_{2}(N)$ and $f(K)=$ $f(N)$, then $K=N$.

(4) For any $0 \neq N \leq M$ and for any nonzero $f \in \operatorname{Hom}_{R}(N, M)$, Ker $f \subseteq$ $\delta(N)$ and if there exists $K \leq N$ with $f(K)=f(N)$, then there exists a projective $L \leq^{\oplus} N$ such that $L \subseteq K e r f$ and $N=K \oplus L$.

\section{Proof.}

(1) $\Rightarrow(2)$ Suppose that $M$ is a $\delta$-small monoform module. Let $0 \neq N \leq M$ and $0 \neq f \in \operatorname{Hom}_{R}(N, M), f \neq 0$. Let $K \leq N$ such that $f(K)=f(N)$. Thus, $K \operatorname{erf}+K=N$. Since $M$ is a $\delta$-small monoform module, $\operatorname{Ker} f \ll \delta$ $N$. By Lemma 1.4, $N=K \oplus L$ for some projective semi-simple submodule $L$ with $L \subseteq K \operatorname{Kerf}$. This implies that $N / K \cong L$. Since $\left(K+Z_{2}(N)\right) \leq_{e} N$, $N / K$ is Godie torsion by ([2], Proposition 2.2). Thus, $N / K \cong L$ is Goldie torsion. Therefore, $L=\{0\}$, i.e., $N=K$.

$(2) \Rightarrow(1)$ Let $0 \neq N \leq M$ and $f: N \longrightarrow M$ be a nonzero homomorphism. Suppose $\operatorname{Kerf}+K=N$ with $N / K$ singular for some $K \leq N$. It is clear that $N / K$ is Goldie torsion. So, in view of Proposition 2.2 in [2], we have $\left(K+Z_{2}(N)\right) \leq_{e} N$. Therefore, $N=K$.

$(2) \Leftrightarrow(3)$ Follows from Proposition 2.2 in [2].

$(1) \Leftrightarrow(3)$ According to the argument used in the proof of $(1) \Rightarrow(2)$ and in view of Proposition 2.2 in [2], this equivalence holds true.

$(1) \Rightarrow(4)$ It is clear.

(4) $\Rightarrow(1)$ Let $0 \neq N \leq M$ and $f: N \longrightarrow M$ be a nonzero homomorphism. Suppose $\operatorname{Kerf}+K=N$ with $N / K$ singular for some $K \leq N$. This implies that

$f(N)=f(K)$. Then by hypothesis, there is a projective $L \leq^{\oplus} N$ with $L \subseteq K \operatorname{Ker} f \subseteq \delta(N)$ such that $N=K \oplus L$. Hence $\delta(L) \oplus \delta(K)=\delta(N)=$ $L \oplus \delta(N)$. Thus $\delta(L)=L$. Since $L$ is projective, $L$ is semi-simple by Proposition 2.13 in [9]. Thus $N / K \cong L=\{0\}$, i.e, $N=K$. Therefore, $M$ is a $\delta$-small monoform module.

Corollary 3.18. Every $\delta$-small module which does not have any nonzero projective semi-simple is a small monoform module.

\section{Proof.}

Let $M$ be a $\delta$-small module, $N$ be a nonzero submodule of $M$ and $f$ : $N \longrightarrow M$ be a nonzero homomorphism. Assume $\operatorname{Kerf}+K=N$ for some $K \leq N$. Since $M$ is assumed $\delta$-small monoform, $\operatorname{Kerf} \ll_{\delta} N$. Then by 
Theorem 2.17, $N=K \oplus L$ for some projective semi-simple submodule $L$ with $L \subseteq K \operatorname{erf}$. By hypothesis, $L=\{0\}$. This implies that $N=K$ and so $\operatorname{Kerf} \ll N$. Hence, $M$ is a small monoform module.

Corollary 3.19. The following statements are equivalent for a uniform $R$ module $M$ :

(1) $M$ is a $\delta$-small monoform module.

(2) $M$ is an e-small monoform module.

(3) $M$ is a small monoform module.

Proposition 3.20. Let $M$ be a free $\mathbf{Z}$-module. Then the following conditions are equivalent:

(1) $M$ is an e-small monoform.

(2) $M$ is a $\delta$-small monoform.

Proof. $(1) \Rightarrow(2)$ Suppose $M$ is an $e$-small monoform module. Let $0 \neq$ $N \leq M$ and $f: N \longrightarrow M$ be a nonzero Z-homomorphism. Suppose $K \operatorname{erf}+K=N$ with $N / K$ singular for some $N \leq K$. For any complement $L$ to $K$ in $N$, we have $K \oplus L \leq_{e} N$. It is clear that $K+L+K e r f=N$. So $K \oplus L=N$ because $\operatorname{Kerf} \ll_{e} N$. By Theorem 2.17, it is easy to see that $K$ is a semi-simple submodule. Since $M$ is a free $\mathbf{Z}$-module, $N$ is also a free $\mathbf{Z}$-module. Hence $N$ is a projective $\mathbf{Z}$-module $\mathbf{I t}$ follows that $K$ is projective. Now, $K$ is a projective semi-simple module. Thus, according to Theorem 2.17, $N=K$. Therefore, $M$ is a $\delta$-small monoform module.

$(2) \Leftarrow(1)$ It is easy to see.

Corollary 3.21. Let $M$ be a projective $\mathbf{Z}$-module. Then the following conditions are equivalent:

(1) $M$ is an e-small monoform.

(2) $M$ is a $\delta$-small monoform.

Proof.

It is clear.

In the following, we give a characterization of small monoform modules.

Proposition 3.22. The following statements are equivalent for an $R$-module $M$ : 
(1) $M$ is a small monoform module.

(2) For any $0 \neq N \leq M$ and for any nonzero $f \in \operatorname{Hom}_{R}(N, M)$, Ker $f \subseteq$ $\operatorname{Rad}(N)$ and if there exists $K \leq N$ with $f(K)=f(N)$, then there exists a projective $L \leq{ }^{\oplus} N$ such that $L \subseteq K \operatorname{Kerf}$ and $N=K \oplus L$.

\section{Proof.}

$(1) \Rightarrow(2)$ It is clear.

(2) $\Rightarrow(1)$ Let $0 \neq N \leq M$ and $f: N \longrightarrow M$ be a nonzero homomorphism. Suppose $\operatorname{Ker} f+K=N$ for some $K \leq N$. By hypothesis, there exists a projective direct summand $L$ of $N$ with $L \subseteq \operatorname{Kerf} \subseteq \operatorname{Rad}(N)$ such that $N=K \oplus L$. So $\operatorname{Rad}(L) \oplus \operatorname{Rad}(K)=L \oplus \operatorname{Rad}(K)$. This implies that $\operatorname{Rad}(L)=L$. Thus, $L=\{0\}$ because $L$ is projective. Hence $K=N$. Therefore, $M$ is a small monoform module.

Definition 3.23. Let $M$ be an R-module.

(1) $M$ is called prime if $A n n_{R}(M)=A n n_{R}(N)$ for each $0 \neq N \leq M$.

(2) $M$ is called compressible if it can be embedded in each of its nonzero submodules.

Remark 3.24. Every compressible $R$-module is a $\delta$-small monoform see [10, Corollary 26.3.5]. The converse is not true in general; for example; the $\mathbf{Z}$-module $\mathbf{Q}$ is a $\delta$-small monoform. But it is not compressible since $\operatorname{Hom}_{\mathbf{Z}}(\mathbf{Q}, \mathbf{Z})=\{0\}$.

Proposition 3.25. Let $M$ be a $\delta$-small monoform semi-simple $R$-module. Then every proper submodule of $M$ is nonsingular.

\section{Proof.}

Let $M$ be a $\delta$-small monoform semi-simple module and $N$ be any proper submodule of $M$. Then $N \mathbb{E}_{e} M$ because $M$ is semi-simple. If $N=\{0\}$, then $N$ is nonsingular. Suppose $N \neq\{0\}$. So, there exists a relative complement $K$ of $N$ in $M$ such that $N \oplus K \leq_{e} M$. Let $f: N \oplus K \longrightarrow M$ define by $f(n+k)=k$ for all $n+k \in N \oplus K$. It is clear that $f$ is welldefined and $f \neq 0$. Since $M$ is $\delta$-small monoform, $\operatorname{Ker} f \ll_{\delta} N \oplus K$. But $\operatorname{Kerf}=N$. Thus, $N \oplus\{0\} \ll_{\delta} N \oplus K$. So according to Lemma 2.3 in [11], $N$ is projective semi-simple. Hence, $N$ is nonsingular.

Corollary 3.26. Let $M$ be a $\delta$-small monoform semi-simple $R$-module. Then any surjection from a $C S R$-module to a proper submodule of $M$ splits. 
Proof. Suppose $M_{1}$ is a $C S$ module and $N$ is a proper submodule of $M$. By Proposition 2.25, $N$ is nonsingular. Let $f: M_{1} \longrightarrow N$ be a surjection. Since $M_{1}$ is a $C S$ module, there exists a direct summand $K$ of $M_{1}$ such that $K \operatorname{erf} \leq_{e} K$. Then $K / K \operatorname{erf}$ is a singular module and it injects into $M_{1}$. Therefore, $K / K \operatorname{erf}=\{0\}$, i.e, $K \operatorname{erf}=K$. This implies that $K \operatorname{erf}$ is a direct summand of $M_{1}$.

Corollary 3.27. Let $M$ be a $\delta$-small monoform semi-simple $R$-module. If $M$ is compressible, then $M$ is nonsingular. In this case, $M$ is isomorphic to a nonzero ideal of a $R$.

\section{Proof.}

In view of Proposition 2.25, every nonzero proper submodule $N$ of $M$ is nonsingular. Since $M$ is a compressible, there exists a monomorphism $f$ : $M \longrightarrow N$. Thus, $f(Z(M)) \subseteq Z(N)$. Since $N$ is nonsingular, $Z(N)=\{0\}$. Thus, $f(Z(M))=\{0\}=f(\{0\})$. Since $f$ is a monomorphism, $Z(M)=\{0\}$. Therefore, $M$ is a nonsingular module. Thus, in view of Theorem 26.4.3 in [12], $M$ is isomorphic to a nonzero ideal of a $R$.

Corollary 3.28. Let $M$ be a $\delta$-small monoform semi-simple $R$-module and $N$ be a proper submodule of $M$. If $K$ and $L$ are two injective submodules of $N$, so is $K+L$.

\section{Proof.}

Let $N$ a proper submodule of $M$. Consider the naturel surjection $f$ : $K \oplus L \longrightarrow K+L$. By Proposition 2.25, $N$ is nonsingular. So, $K+L \subseteq N$ is also nonsingular. Since $K \oplus L$ is injective, it is a $C S$ module. According to the Corollary, $f$ splits. Therefore, $K+L$ is isomorphic to a direct summand of $N \oplus L$, so it is injective.

Corollary 3.29. Let $R$ be a self-injective ring and $M$ be a $\delta$-small monoform semi-simple $R$-module with finite uniform dimension. Then for any proper submodule $N$ of $M, \operatorname{End}_{R}(N)$ is a $C S$ ring.

\section{Proof.}

Let $N$ be a proper submodule of $M$. Then, according to Proposition 2.25, $N$ is a nonsingular module and has finite uniform dimension. Since $R$ is assumed self-injective, $N$ is a $C S$ module by ([8], Exerc.). Since $M$ is semisimlpe, $N$ is also semi-simple. Therefore, according to Theorem 3.2 in [7], $\operatorname{End}_{R}(N)$ is a $C S$ ring. 
Proposition 3.30. The following statements are equivalent for a singular semi-simple $R$-module $M$ :

(1) $M$ is a $\delta$-small monoform module.

(2) $M$ is a monoform module.

(3) $M$ is a simple module.

(4) $M$ is a compressible module.

\section{Proof.}

(2) $\Leftrightarrow(3)$ Follows from Remark 1.3 in [7].

$(1) \Rightarrow(2)$ Suppose $M$ is a $\delta$-small monoform module. Since $M$ is assumed semi-simple, every proper submodule of $M$ is nonsigular by Proposition 2.25. Since $M$ is singular, every proper submodule of $M$ is also singular. From this, every proper submodule of $M$ must be zero. Thus, $M$ is simple module. Therefore, $M$ is a monoform module.

$(2) \Rightarrow(1)$ It is clear.

$(1) \Leftrightarrow(4)$ By argument used in the proof of $(1) \Rightarrow(2)$ and in view of Remark, this equivalence holds true.

Lemma 3.31. Any $\delta$-small monoform singular $R$-module is a uniform module.

\section{Proof.}

Let $M$ be a $\delta$-small monoform singular module. Suppose there exists a nonzero submodule $N \leq M$ such that $N \leq_{e} M$. So, there exists a relative complement $K$ of $N$ in $M$ such that $N \oplus K \leq_{e} M$. Let $f: N \oplus K \longrightarrow M$ define by $f(n+k)=n$ for all $n+k \in N \oplus K$. It is clear that $f$ is well-defined and $f \neq 0$. Since $M$ is $\delta$-small monoform, $\operatorname{Kerf}=\{0\} \oplus K \ll_{\delta} N \oplus K$. In view of Proposition 2.25, $K$ is nonsingular. Since $M$ is singular, $K$ is also singular. From this, $K$ must be zero. This implies that $N \leq_{e} M$. This is a contradiction. Therefore any $\delta$-small monoform singular module is a uniform module.

Theorem 3.32. Let $R$ be an artinian principal ideal ring and $M$ be a singular prime $R$-module. Then the following statements are equivalent:

(1) $M$ is a $\delta$-small monoform module.

(2) $M$ is a monoform module.

(3) $M$ is a small monoform module.

(4) $M$ is a compressible module.

(5) $M$ is a uniform module.

(6) $M$ is a weakly co-Hopfian module. 


\section{Proof.}

$(1) \Rightarrow(2)$ Suppose $M$ is a $\delta$-small monoform module. Let $0 \neq N \leq M$ and $f: N \longrightarrow M$ be a homomorphism. Since $M$ is assumed singular, it is a uniform module by Lemma 2.31. Thus, $M$ is a weakly co-Hopfian module. Since $R$ is an artinian principal ideal ring, $M$ is a finitely generated module by Theorem 3.8 in [3]. So, there exists an epimorphism $g: R \longrightarrow M$ such that $R / \operatorname{ann}_{R}(M) \cong M$. Since $M$ is a prime module, $a n n_{R}(M)$ is a prime ideal of $R$. Hence, $\operatorname{ann}_{R}(M)$ is a maximal ideal of $R$ because $R$ is artinian. This implies that $M$ is a simple module. Hence, $M$ is a monoform module.

$(2) \Rightarrow(1)$ It is clear.

$(1) \Leftrightarrow(3))$ It is easy to see.

$(4) \Rightarrow(1)$ Results from Remark 2.24.

$(1) \Rightarrow(4)$ According to the argument used in the proof of $(1) \Rightarrow(2)$, we get that $M$ is a uniform prime finitely generated module. Therefore, in view of Lemma 26.2.9 in [10], $M$ is a compressible module.

$(2) \Rightarrow(5)$ It is clear.

$(5) \Rightarrow(2)$ Suppose $M$ is a uniform module. According to the proof of

$(1) \Rightarrow(2), M$ is a simple module. Therefore, $M$ is a monoform module.

$(5) \Rightarrow(6)$ is clear.

$(6) \Rightarrow(5)$ Suppose $M$ is a weakly co-Hopfian module. Then, $M$ is a simple module. Therefore, $M$ is a uniform module.

Remark 3.33. It is clear that a simple module is a $\delta$-small monoform prime module. But the converse is not true in general. For example $\mathbf{Z}$ as $\mathbf{Z}$-module is a delta-small monoform prime module. However, it is not simple.

Corollary 3.34. Let $R$ be an artinian principal ideal ring. Then the following statements are are equivalent for a singular $R$-module $M$ :

1. $M$ is a $\delta$-monoform prime module.

2. $M$ is a simple module.

Proof. $\mathrm{F}(1) \Rightarrow(2)$. Suppose $M$ is a $\delta$-monoform prime module. Then $\operatorname{ann}_{R}(M)$ is a prime ideal of $R$. According to Theorem 2.32, $M$ is a simple module.

$(2) \Rightarrow(1)$ Follows from Remark 2.33. 
Corollary 3.35. Let $R$ be an artinian principal ideal ring. Then the following assertions are verified for a singular $\delta$-small monoform $R$-module:

1. $\operatorname{End}_{R}(M)$ is a local ring.

2. $M$ is a direct sum of a semi-simple module $N$ and a module $K$ such that $\delta(K) \leq_{e} K$.

\section{Proof.}

1. Since $M$ is a finitely generated module over an artinian ring, $M$ is of finite length. Thus, $M$ is an indecomposable module of finite because $M$ is a uniform module. Therefore, $\operatorname{End}_{R}(M)$ is a local ring.

2. For $\delta(M)$, there exists $N \leq M$ such that $N \cap \delta(M)=\{0\}$ and $N \oplus \delta(M) \leq_{e} M$. According to 1$), M$ is a finitely generated module over an artinian ring. Thus, in view of Example 2.25 in [11], there exists $K \leq M$ such that $N+K=M$ and $N \cap K \ll_{\delta} K$. Since $N \cap K=N \cap(N \cap K) \leq N \cap \delta(K) \leq N \cap \delta(M)=\{0\}, M=N \oplus K$. This implies that $N$ is a semi-simple module. The last part is clear because $N \oplus \delta(K) \leq_{e} N \oplus K$.

For an $R$-module the dual module $\operatorname{Hom}_{R}(M, R)$ is denoted by $M^{*}$. If the natural map $M \longrightarrow\left(M^{*}\right)^{*}$ is injective, then $M$ will be called torsionless. $M$ is called reflexive if the natural map $M \longrightarrow\left(M^{*}\right)^{*}$ is bijective.

Proposition 3.36. Let $R$ be a quasi-Frobenius principal ideal ring and $M$ be a $\delta$-small monoform singular $R$-module. Then the following assertions are verified:

1. $M$ is reflexive.

2. $M^{*}$ and $E\left(M^{*}\right)$ are finitely generated.

\section{Proof.}

1. In view of Theorem $2.32, M$ is a finitely generated $R$-module. Therefore, accordind to Theorem 15.11 in [8], $M$ is reflexive.

2. Since $M^{*}$ is finitely generated and $A$ is artinian, $E\left(M^{*}\right)$ is finitely generated.

Proposition 3.37. Let $M$ be a $\delta$-small monoform $R$-module and $f$ a surjecive endomorphism of $M$. If $N \leq M$, then $f(N) \ll_{\delta} M$ if and only if $N \ll_{\delta} M$. 
Proof. $\Rightarrow)$ Let $N+B=M$ with $M / B$ singular for some $B \leq M$. Then $f(N)+f(B)=M$. Then $f(B)=M$ because $f(N) \ll_{\delta} M$ and $M / f(B)$ singular. This implies that $\operatorname{Kerf}+B=M$. Since $\mathrm{M}$ is a $\delta$-small monoform R-module, $\operatorname{Kerf} \ll_{\delta} M$. Hence $B=M$. Therefore $N \ll_{\delta} M$.

$(\Leftarrow$ holds true from Lemma 1.5.

Definition 3.38. An $R$-module is called fully retractable if for every nonzero submodule $N$ of $M$ and every nonzero element $g \in H_{0}(N, M)$, we have $\mathrm{Hom}_{R}(M, N) g \neq\{0\}$.

Proposition 3.39. Let $M$ be a fully retractable $R$-module such that for each

$0 \neq N \leq M$, the kernel of any nonzero endomorphism of $N$ is $\delta$-small.

Then $M$ is a $\delta$-small monoform module.

Proof. Let $0 \neq N \leq M$ and $f: N \longrightarrow M$ such that $f \neq 0$. Since $M$ is fully retractable, there exists $g: M \longrightarrow N, g \neq 0$. Consider $N \stackrel{f}{\longrightarrow} M \stackrel{g}{\longrightarrow} N$. We have $g \circ f \neq 0$ because $M$ is fully retractable. By hypothesis, $\operatorname{Ker}(g \circ$ $f) \ll_{\delta} N$. Since $\operatorname{Ker} f \subseteq \operatorname{Ker}(g \circ f)$, thus by Lemma 1.5, $\operatorname{Ker} f \ll_{\delta} N$. So $M$ is an $\delta$-small monoform module.

Definition 3.40. A submodule $N$ of a module $M$ is called $\delta$-coclosed if $N / K$ is singular and $N / K \ll_{\delta} M / K$ for some $K \leq N$ implies $K=N$

Proposition 3.41. Let $M$ be a quasi-injective $R$-module such that every submodule of $M$ is $\delta$-coclosed. If the kernel of any nonzero endomorphism of $M$ is $\delta$-small, then $M$ is a $\delta$-small monoform.

Proof. $\Rightarrow$ Let $0 \neq N \leq M$ and $f \in \operatorname{Hom}_{R}(N, M), f \neq 0$. Since $M$ is quasi-injective, there exists $g \in \operatorname{End}_{R}(M)$ such that $g \circ i=f$ where $i$ is the inclusion map. Hence, $g(n)=f(n)$ for each $n \in N$ and so Kerf $\leq K e r g$. By hypothesis, $\operatorname{Kerg} \ll_{\delta} M$. So Kerf $\ll_{\delta} M$. On the other hand, Kerf $\leq$ $N$ and by [4, Corollary 2.6], Kerf $\ll_{\delta} M$ implies $K \operatorname{erf} \ll_{\delta} N$. Therefore, $M$ is a $\delta$-small monoform module.

$(\Leftarrow \mathrm{It}$ is clear. 


\section{References}

[1] M. S. Abbass, "On fully stable modules", Ph. D. Thesis, University of Baghdad Iraq, 1990.

[2] S. Asgari and A. Haghany, "Generalizations of t-extending modules relative to fully invariant submodules", Journal of the Korean Mathematical Society, vol. 49, no. 3, pp. 503-514, May 2012, doi: 10.4134/JKMS.2012.49.3.503

[3] M. Barry and P.C. Diop, "Some properties related to commutative weakly FGI-rings", JP Journal of algebra, number theory and application, vol. 19, no. 2, pp. 141-153, Dec. 2010. [On line]. Available: https://bit.ly/2VOfw5W

[4] E. Buyukasik and C. Lomp, "When $\delta$-semiperfect rings are semiperfect", Turkish journal of mathematics, vol. 34, pp. 317-324, 2010, doi: 10.3906/mat-0810-15

[5] A. D. Diallo, P. C. Diop, and M. Barry, "On e-small monoform modules", JP Journal of algebra, number theory and application, vol. 40, no. 3, pp. 305-320, Jun. 2018. [On line]. Available: https://bit.ly/310QJlY

[6] M. A. Inaam Hadi and K. H. Marhoon, "Small monoform modules", Ibn AL- Haitham journal for pure and applied science, vol. 27, no. 2, pp. 229240, Aug. 2014. [On line]. Available: https://bit.ly/3f7IIfK

[7] S. M. Khury, "Nonsingular retractable modules and their endomorphism rings", Bulletin of the Australian Mathematical Society, vol. 43, no. 1, pp. 63-71, Feb. 1991, doi: 10.1017/S000497270002877X

[8] T. Y. Lam, Lectures on modules and rings, New York, NY: Springer, 1999, doi: 10.1007/978-1-4612-0525-8

[9] A. Ç. Özcan and M. Alkan, "Semiperfect modules with respect to a preradical," Communications in algebra, vol. 34, no. 3, pp. 841-856, Feb. 2006, doi: 10.1080/00927870500441593 
[10] P. F. Smith, "Compressible and related modules", in Algebra groups, rings, modules and homological algebra, P. Goeteres and O.M.G Jenda, Boca Raton, FL: Chapman \& Hall/CRC, 2006, doi: 10.1201/9781420010763

[11] R. Tribak, "Finitely generated $\delta$-supplemented modules are amply $\delta$ supplemented", Bulletin of the Australian Mathematical Society, vol. 86, no. 2, pp. 430-439, Feb. 2012, doi: 10.1017/S0004972711003406

[12] J. M. Zelmanowitz, "Representation of rings with faithful polyform modules", Communications in algebra, vol. 14, no. 6, pp. 1141-1169, 1986, doi: 10.1080/00927878608823357

[13] Y. Q. Zhou, "Generalization of perfect, semiperfect and semiregular rings", Algebra colloquium, vol. 7, pp. 305-318, Aug. 2000, doi: 10.1007/s10011-000-0305-9 